\title{
La atencion a la diversidad en el sur de España: concepciones y práctica del pedagogo terapeuta
}

\author{
Attention to diversity in southern spain: conceptions and practice of \\ the therapist pedagogue
}

\author{
Manuel Delgado García* \\ Universidad de Sevilla \\ Francisco Javier García Prieto** \\ Universidad de Huelva
}

Resumo Esta investigación tiene como objetivo profundizar en la realidad del planteamiento de la atención a la diversidad en la etapa de educación primaria. A través de un enfoque cualitativo, se analizan las concepciones y prácticas que desarrolla el profesorado de pedagogía terapéutica (PT) en sus aulas; los avances y limitaciones que experimenta; el tipo de formación continua de la que disponen, entre otras. Los resultados muestran el grado de inclusión de sus acciones a la vez que indican la necesidad de fomentar la implicación entre docentes y mejorar la formación de los especialistas. Las conclusiones indican la satisfacción del PT con su práctica educativa y la necesidad de una formación continua que ayude a buscar el desarrollo normalizado del estudiante y la superación de las barreras existentes.

PALAVRAS-CHAVE: Atención a la diversidad; Formación del profesorado; Educación inclusiva.

\begin{abstract}
The objective of this research is to deepen in the reality of the diversity attention in primary education stage. From a qualitative approach, the conceptions and practices developed by therapeutic pedagogy (PT) teachers in their classrooms are analyzed. Besides, the advances and limitations that they experience and the type of their continuous training, among others, are also considered. The results show the degree of inclusion of their actions, while indicating the need to foster involvement among teachers and improve the training of specialists. The conclusions indicate the PT's satisfaction with its educational practice and the need for continuous training that helps to seek the student's standard development and overcoming existing barriers.
\end{abstract}

KEYWORDS: Attention to diversity; Teacher training; Inclusive education. 


\section{Introducción}

La educación inclusiva surge para sustituir al concepto de integración; a raíz de ahí, son numerosos los trabajos que tratan de definirlo (AINSCOW, 2004, 2010; BARTON, 2008; BOOTH, 2009; ECHEITA y VERDUGO, 2005; ESCUDERO y MARTÍNEZ, 2011; GLAT, PLETSCH y FONTES, 2007; LÓPEZ-MELERO, 2012; SÁNCHEZ-TERUEL y ROBLES-BELLO, 2013) y, de todos ellos se podría configurar una definición que referencie a la educación inclusiva como un término desde el que se promueve la igualdad de oportunidades socioeducativas y culturales para todas las personas, partiendo de sus capacidades particulares y niveles de logro, atendiendo a la diversidad existente, para alcanzar una formación integral.

También se extrae la idea de que la inclusión, a nivel teórico, es asumida de forma universal, pero en la mayoría de casos, carece de mecanismos que permitan su irrupción de manera efectiva en las escuelas (ARNAIZ y GUIRAO, 2015), de ahí la necesidad de "hacer las escuelas más inclusivas" (AINSCOW, BOOTH y DYSON, 2006; AINSCOW, 2012) ante los nuevos desafíos de la educación en el siglo XXI. Se trata de superar las diferentes "barreras (políticas: normas contradictorias; culturales: conceptuales y procedimentales; didácticas: enseñanza-aprendizaje) que impiden la participación, la convivencia y el aprendizaje en la escuela" (LÓPEZ-MELERO, 2012, p.143) de aquellos niños y niñas a los que no se respetan sus diferencias. Se trata de una actitud común y de un compromiso comunitario que contribuya a una educación de calidad para todo el alumnado. Alcanzar esta meta supone afrontar un proceso progresivo que implica, tanto un cambio sistémico del sistema educativo (estructura, financiación, currículo, la organización y funcionamiento de los centros, el modelo de orientación educativa y psicopedagógica, etc.), como un cambio en la concepción del profesorado, pues los aspectos epistemológicos y metodológicos inciden en el tipo de intervención educativa que se desarrolla (ALONSO y ARAOZ, 2011; ECHEITA et al., 2013).

Es en esta segunda variable donde este trabajo centra su atención puesto que, en el contexto español existe un estancamiento en cuanto a apoyos institucionales para atender a la diversidad, traduciéndose en una falta de competencias y de motivación para afrontar las numerosas funciones que atiende el profesorado (ESCUDERO y MARTÍNEZ, 2011; ECHEITA et ál., 2009; SANDOVAL, SIMÓN y ECHEITA, 2012). Unas funciones ligadas a la atención del alumno con Necesidades Específicas de Apoyo Educativo (NEAE) y asociadas a la labor de "apoyo" ejercida por varias figuras profesionales (profesorado de Pedagogía Terapéutica-PT-, de Audición y Lenguaje -AL-o de Compensatoria), en vez de afrontarse como un proceso compartido por el centro educativo.

En concreto, nos aproximamos a la figura del especialista en Pedagogía Terapéutica quien, a partir de la normativa vigente (Gráfico nº1), es el responsable de atender a la diversidad en colaboración con el resto de docentes y cuya labor se desempeña (ANGULO et al, 2008):

Dentro aula ordinaria: es la opción más inclusiva, permaneciendo en el aula junto al profesorado y todo el alumnado (máximo 3 alumnos/as con NEAE). 
Aunque existen otras posibilidades que le llevan a participar:

En aula ordinaria y apoyos en periodos variables: opción muy frecuente ante el amplio número de alumnado con NEAE; el alumnado (máximo 5 alumnos/as con NEAE por sesión y de 15 a 20 en total) sale del aula ordinaria para recibir apoyo a través de diversos programas en el Aula de Apoyo a la Integración (atención individualizada o pequeños grupos).

Dentro del aula específica: el alumnado es atendido de manera individualizada en pequeños grupos y salen del aula para asistir al aula ordinaria en aquellas materias que puede seguir junto a su grupo de referencia (el número es variable: Psíquicos: 6-8; Sensoriales: 6-8; Físicos-motóricos: 8-10; Autistas o psicóticos: 3-5; Plurideficientes: 4-6; Unidades que escolarizan alumnado de diferentes discapacidades: 5 ).

Gráfico 1 - Resumen de la normativa que regula las funciones del PT en Andalucia.

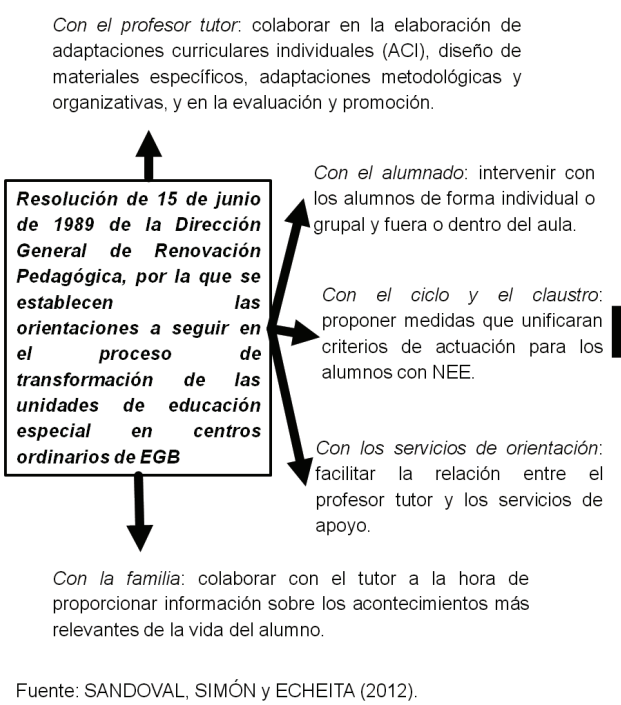

Artículo 19: «La atención al alumnado con NEE escolarizado en grupos ordinarios con apoyos en periodos variables se llevará a cabo, preferentemente, en dicho grupo».

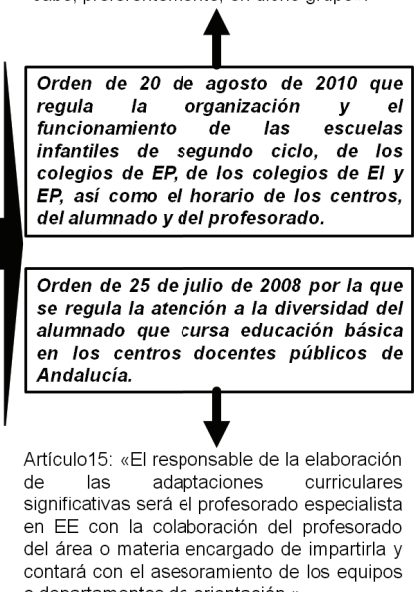
contará con el asesoramiento de los equipos o departamentos de orientación

En concreto, desarrolla diferentes medidas para atender a la diversidad: " $d e$ carácter curricular" ya sean puntuales o permanentes (refuerzo educativo, adaptaciones curriculares o programas de diversificación curricular) y, "de carácter organizativo" para flexibilizar la enseñanza a partir de la autonomía de los centros (integración de materias en ámbitos, agrupamientos flexibles, desdoblamientos de grupos, oferta de materias optativas, programas de refuerzo, programas de tratamiento personalizado).

Así pues, se entiende que la gestión estratégica de la diversidad por parte de este especialista es un factor esencial para darlo a conocer a la comunidad educativa y, de ahí que existan trabajos de gran interés que enfatizan las percepciones del profesorado sobre su propia acción docente en relación a la inclusión o a la atención 
a la diversidad (ÁLVAREZ et al., 2009; BOOTH y AINSCOW, 1998; FLORIAN y BLACK-HAWKINS, 2011; LLEDÓ y ARNAIZ, 2010; MUNTANER, 2014; ROSE, 2001; LOPES et al, 2004; SCHAPER, 2010).

Siguiendo esta línea de trabajo, la investigación que se presenta tiene como objetivo dar a conocer la percepción de los maestros de PT con respecto a la situación de la atención a la diversidad en la provincia de Sevilla. Para ello, se presta especial atención a:

Describir cómo se entiende la intervención docente especialista (estrategias didácticas, apoyos, etc.).

Mostrar el funcionamiento de las aulas (organización, clima, etc.) donde se atiende a la diversidad.

Exponer las potencialidades y barreras existentes (resultados, obstáculos, etc.) en las prácticas educativas inclusivas.

\section{Diseño de investigación}

Se llevó a cabo una investigación con un planteamiento cualitativo de tipo descriptivo-interpretativo. El objeto de estudio queda claramente individualizado al examinar la realidad desde los significados, sentimientos, prácticas, creencias y valores que los protagonistas experimentan en los escenarios de aula.

\section{Participantes}

Se aborda un universo de análisis particular conformado por el profesorado especialista en PT que participa, durante el curso 2015/2016, en los procesos de integración e inclusión del alumnado con NEAE en los Centros de Educación Infantil y Primaria (CEIP) de la provincia de Sevilla (España). De una población total de 406 CEIP y mediante un muestreo aleatorio simple, se seleccionan 48 centros (representatividad del 11,82\%) con su respectivo profesional en PT. De estos 48 participantes, el $87,3 \%$ son mujeres y el 12,7\% hombres. La media de antigüedad es de 11,2 años.

El estudio, además, comprende la oferta formativa (planes de formación) que los 6 Centros de Profesores (CEP) de la provincia de Sevilla ponen al servicio de los docentes de educación primaria, en los cursos 2015/2016 y 2016/2017 con respecto al ámbito de la atención a la diversidad.

\section{Técnicas para la recogida y el análisis de la información}

Para bordar el objeto de estudio se recurre a tres técnicas concretas:

- Entrevista semiestructurada: Diseñada ad hoc, se compone de 18 ítems de respuesta abierta, validados a través de la técnica juicio de expertos y mediante una prueba piloto (2 especialistas en PT en un CEIP de Sevilla).

- Análisis de contenido: a través de una lista de control (Tabla nº1), se procede a vertebrar los planes de formación docente en unidades de signifi- 
cación y de registro que contabilizan y categorizan las iniciativas formativas. La confiabilidad y validez de este tipo de análisis se asegura a través de la coherencia interna que aporta el uso de este material a la investigación, así como la garantía de exhaustividad, representatividad, homogeneidad, pertinencia y univocación que se ha dado al mismo.

Tabela 1 - Lista de control para el análisis de los Planes de Formación de los CEP.

\begin{tabular}{|c|c|c|c|c|}
\hline $\begin{array}{c}\text { Nombre del } \\
\text { CEP }\end{array}$ & $\begin{array}{l}\text { Modalidad de } \\
\text { impartición }\end{array}$ & Destinatarios & Temáticas/Descriptores & $\begin{array}{c}\text { Título de la } \\
\text { actividad }\end{array}$ \\
\hline $\begin{array}{l}\text { 1.Sevilla } \\
\text { 2.Castilleja de } \\
\text { la Cuesta } \\
\text { 3.Écija-Osuna } \\
\text { 4.Alcalá de } \\
\text { Guadaira } \\
\text { 5.Lebrija } \\
\text { 6. Lora del Río }\end{array}$ & $\begin{array}{l}\text { - Conferencia } \\
\text { - Congreso } \\
\text { - Curso } \\
\text { - Curso a distancia } \\
\text { - Curso con } \\
\text { seguimiento } \\
\text { - Curso } \\
\text { semipresencial } \\
\text { - Formación en } \\
\text { centros } \\
\text { - Encuentro } \\
\text { - Formación } \\
\text { específica en } \\
\text { centros } \\
\text { - Grupo de trabajo } \\
\text { - Jornadas }\end{array}$ & $\begin{array}{l}\text { Educación } \\
\text { infantil y } \\
\text { primaria }\end{array}$ & $\begin{array}{l}\text { - Atención al alumnado } \\
\text { de carácter } \\
\text { compensatorio } \\
\text { - Atención al alumnado } \\
\text { con dificultades de } \\
\text { aprendizaje } \\
\text { - Atención al alumnado } \\
\text { con Necesidades } \\
\text { Educativas Especiales } \\
\text { - Otras }\end{array}$ & \begin{tabular}{|l|} 
Ejemplo: \\
Jornadas de \\
escuela \\
inclusiva: una \\
apuesta por \\
el éxito \\
educativo \\
\end{tabular} \\
\hline
\end{tabular}

Fuente: elaboración propia (2016).

- Observación: a través de un registro observacional representado en el diario de los investigadores, se identifican aquellas situaciones derivadas de las 48 entrevistas que facilitan la interpretación de la información recopilada.

Las entrevistas se realizan de manera individual (aula específica del centro), previo acuerdo telefónico con el informante, y con una duración media de $90 \mathrm{minu}-$ tos. Finalmente, para el proceso de análisis se utiliza el Atlas Ti (versión 6.2), en el que se configura una unidad hermenéutica. Se diseña un sistema de categorías (Tabla $\left.n^{\circ} 2\right)$ que segmenta y codifica la información; en concreto, las citas textuales utilizadas mantienen el siguiente diseño: ejemplo: "Ajustar el currículo a las necesidades de cada alumno" (6:45, 123:401, D6), significando: 6:45 = número de entrevista: número de cita en dicho documento, 123:401 = párrafo donde se inicia la cita: párrafo donde termina la cita. D6: docente que realiza la afirmación. 
Tabela 2 - Sistema categorial.

\begin{tabular}{|c|c|c|}
\hline DIMENSIONES & CATEGORIAS & ÍTEMS \\
\hline \multirow{7}{*}{$\begin{array}{c}\text { La intervención } \\
\text { docente }\end{array}$} & Formación & $\begin{array}{l}\text { ¿Considera que tiene formación suficiente para atender a la } \\
\text { diversidad en una escuela para todos-as? }\end{array}$ \\
\hline & Percepciones & $\begin{array}{l}\text { - ¿Qué entienden los compañeros por atención a la diversidad? } \\
\text { - ¿Qué opinan de vuestra labor? }\end{array}$ \\
\hline & Estrategias didácticas & $\begin{array}{l}\text { ¿Qué estrategias didácticas se desarrollan para atender a la } \\
\text { diversidad? }\end{array}$ \\
\hline & Apoyos & ¿Apoyo dentro o fuera del aula? ¿por qué? \\
\hline & Dinámica & $\begin{array}{l}\text { - ¿Cómo se lleva a cabo la dinámica cotidiana del aula? } \\
\text { - ¿Qué proceso se sigue? }\end{array}$ \\
\hline & Recursos & - ¿Con qué recursos cuenta y qué recursos concretos se utilizan? \\
\hline & Evaluación & $\begin{array}{l}\text { - ¿Qué resultados se obtienen? } \\
\text { - ¿Cómo se realiza y en qué te fijas para evaluar? }\end{array}$ \\
\hline \multirow{3}{*}{ El aula } & Organización & - ¿Cómo se prepara y organiza el aula? \\
\hline & Clima & $\begin{array}{l}\text { - ¿El alumnado se siente cómodo? } \\
\text { - ¿Cómo es la convivencia? }\end{array}$ \\
\hline & Familia & - ¿En qué medida participa la familia del alumnado? \\
\hline \multirow[t]{3}{*}{$\begin{array}{l}\text { Avances y } \\
\text { limitaciones }\end{array}$} & Medidas & $\begin{array}{l}\text { ¿Qué medidas se han llevado a cabo para una educación sin } \\
\text { exclusiones? } \\
\text { ¿A qué afectan dichas medidas: distribución del profesorado, } \\
\text { horarios, materiales, adaptaciones curriculares, reformulación del } \\
\text { currículum, agrupamientos del alumnado, etc.? }\end{array}$ \\
\hline & Resultados & - ¿Satisfacción, logros y expectativas? \\
\hline & Obstáculos & $\begin{array}{l}\text { - ¿Cuáles son los obstáculos y dificultades que limitan su labor para } \\
\text { atender a la diversidad? }\end{array}$ \\
\hline
\end{tabular}

Para la credibilidad se recurre a criterios de triangulación metodológica y revisión por los interesados, imprimiendo consistencia en las conclusiones obtenidas.

\section{Análisis de resultados}

El proceso se configura mediante el sistema categorial establecido, y los resultados se engloban en base a las dimensiones y categorías mostradas.

\section{La intervención docente}

En el ámbito de la formación, la mayoría del profesorado reconoce tener capacidades suficientes para atender a la diversidad. No obstante, algunos de los participantes (6 docentes) señalan la necesidad de recurrir a la formación continua para mejorar su cualificación profesional: "nosotras, mediante cursos, tenemos que complementar nuestra formación según las características del centro y del alumnado" (3:12, 13:14, D3).

Analizando la formación continua durante los cursos 2015/2016 y 2016/2017, se aprecia cómo "la atención a la diversidad/la escuela inclusiva" aparece como una de las temáticas más relevantes (8\%) junto a tecnologías (16\%), idiomas (13\%), desarrollo de competencias (11\%) o metodologías de enseñanza y aprendizaje (12\%). En el Gráfico n²2, se estructura la tipología de esta formación, de la que destacan acciones como: "curso de tratamiento, intervención y atención al alumnado con TDHA"; "Grupo de trabajo: en la escuela cabemos todos"; "Jornadas de escuela inclusiva: una apuesta por el éxito educativo", "Formación en centros: alumnado ayudante, recurso para la inclusión y prevención de conflictos" etc. 


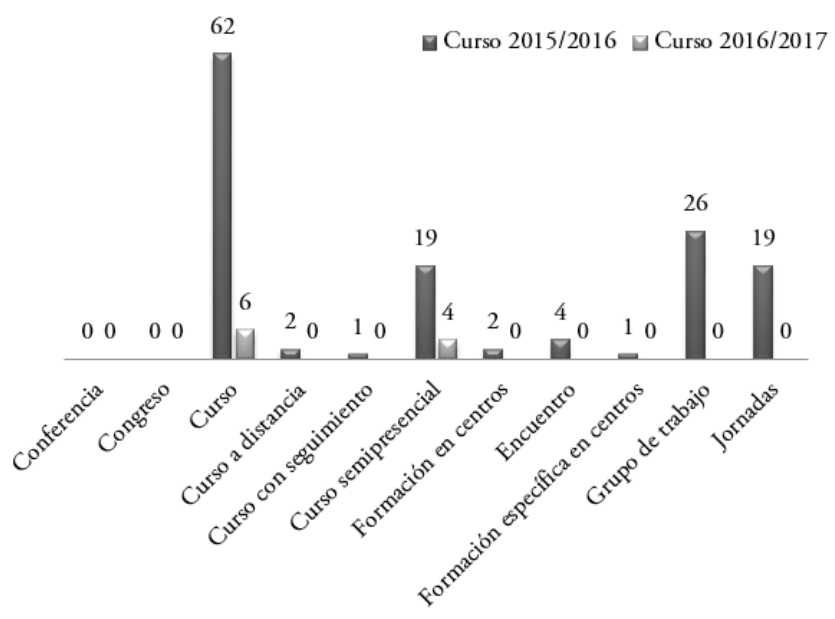

Abordando las percepciones del profesorado respecto al significado, atender a la diversidad se define como "la necesidad de atender a todo el alumnado por igual" (34:21, 18:22, D34); “atender a las necesidades del alumnado de manera individual” (9:64, 6:7, D9) o asociarla con la atención a estudiantes "discapacitados, con dificultades, con problemas, con necesidades educativas diferentes, que necesitan ayuda y que no puede atender el tutor/a, o que no sienten lo mismo que los normales" (43:64, 7:11, D43); esta variedad muestra distintos grados de conocimiento, valoración e implicación del PT en el aula, correspondiéndose con centros en los que la coordinación entre el claustro de profesores es evidente ("la gran mayoría del profesorado es consciente de que la diversidad está presente en las aulas y apostamos por un trabajo cooperativo y por la inclusión en la medida de nuestras posibilidades") $(41: 4,15: 20, \mathrm{D} 41)$ y con otros en los que es inexistente ("En general, no tienen en cuenta ese término en la vida diaria escolar y es difícil contar con el apoyo de los compañeros") (15:8, 23:28, D15).

En lo que respecta a las estrategias didácticas, los resultados proporcionan las siguientes variables:

a) El espacio se diversifica entre el aula ordinaria, los apoyos y las aulas específicas. "A veces el/la alumno/a sale fuera del aula y en otras el docente entra dentro de ella, formando parte activa de la clase y no centrándose en el alumno con NEAE" (28:41, 18:22, D28).

b) Impera la necesidad de establecer un protocolo de actuación previo para atender a la diversidad. Podría estructurarse de la siguiente forma: "a) Partir de la situación del alumno; b) Facilitar la construcción de aprendizajes signifcativos; c) Plantear el aprendizaje como modificación de los esquemas de conocimiento y adecuado a las posibilidades del alumnado; d) Desarrollar una inter- 
vención comunicativa en los contextos menos restrictivos posibles" (4:22, 33:35, D4).

d) Existe un cierto grado de acuerdo a la hora de hablar de medidas ordinarias y extraordinarias como las principales vías para iniciar la atención a todo el alumnado: "Atendemos a la diversidad desde la programación de aula, en ella deben estar incluidas las medidas de carácter ordinario, así como las medidas de carácter extraordinario" (32:10, 79:81, D32). A continuación (Gráfico $\mathrm{n}$ 3), se agrupan las medidas recopiladas:

Gráfico 3 - Medidas para atender a la diversidad.

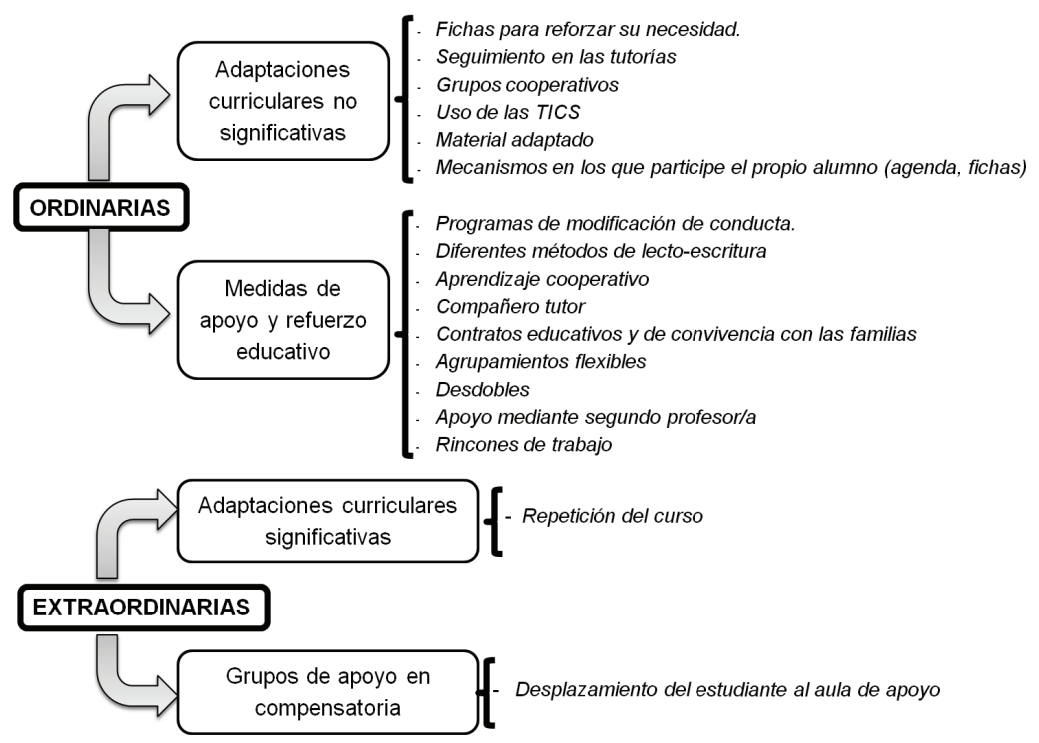

Al profundizar en la forma de afrontar el apoyo (dentro o fuera del aula específica), los informantes hablan de esta dualidad para atender las distintas situaciones a las que se enfrenta el estudiante ("El apoyo debe de darse dentro del aula ordinaria para garantizar una atención lo mas inclusiva posible. Participar de los mismos espacios tiempos y actividades. No obstante, ni el número de alumnos, ni los contenidos de la intervención permiten esta atención") (16:45, 29:31, D16), pero también afirman que la atención a la diversidad dependerá en gran medida de las dificultades que presente el alumno. Hay diferencias entre la atención en la etapa de infantil y la etapa de primaria ("En primaria es fuera del aula y en infantil dentro del aula ya que, como son tan pequeños, la idea es que estén en el aula y alli el PT se encargue de of recerles la atención que necesitan que normalmente es más control conductual') (44:5, 44:48, D44).

También se cuestiona la dinámica seguida durante el desarrollo de una sesión de trabajo con el alumnado, y es preciso diferenciar entre las que se producen en el seno del aula ordinaria y producen fuera de esta (aulas específicas): "A principio de curso organizamos la atención a los/as niños/as con NEAE procurando acompañarlos toda su 
escolaridad (...) procurando hacer grupos lo más homogéneos posibles en cuanto a tipología de alumno, nivel curricular..."(29:76, 23:27, D29).

Así pues, las secuencias descritas para el trabajo en un aula ordinaria coinciden en la siguiente dinámica: "Comenzamos entrando en las aulas y sentándonos en una mesa con los alumnos que necesitan apoyo educativo. Hacemos las tareas que se mandan para todos sus compañeros, si terminan antes podemos hacerles algunas actividades especificas para sus necesidades o, si es necesario, se le hacen más actividades de refuerzo" (30, 48:49, D30).

Las secuencias ligadas a las sesiones en aulas específicas presentan dinámicas con mayor uniformidad en cuanto a los tiempos y la rutina diaria:

- "Las sesiones de trabajo son de 45 minutos y se atiende hasta un máximo de 3-4 alumnos/as. A principio de curso se pone en conocimiento de los/ as tutores/as, familias y alumnado el horario de atención, para que sean conocedores de cuántas sesiones semanales van a contar con nuestro apoyo. Nuestra intervención puede ir desde una atención curricular más individualizada hasta el desarrollo de programas específicos" (12:61, 26:28, D12).

- "Se suelen agrupar a los niños por edades, ciclos o dificultades, exceptuando algunos con los que es necesario trabajar de manera individual, sin otros niños en el aula" (26:59, 30:31, D26).

- "Son sesiones que no coinciden con las asignaturas de especialidades (Educación física, música-artística o inglés), suele atenderse al alumnado en horario de materias instrumentales como lengua o matemáticas" (4:5, 23:24, D4).

- "La dinámica viene marcada por unas rutinas que se repiten a diario: $1^{\circ}$ Bienvenida y asamblea; $2^{\circ}$ Rincones de trabajo; $3^{\circ}$ Desayuno y aseo; $4^{\circ} \mathrm{Re}-$ creo; 5 Relajación; 6 Periodo de integración" (1:32, 56:58, D1).

Los informantes (casi en su totalidad) afirman que disponen de una amplia dotación de recursos para la atención a la diversidad en las aulas, "cuento con muchos recursos que los pone a disposición el propio centro, la Junta de Andalucia y otros recursos elaborados por mi" (45:63, 67:69, D45), pero existen casos en los que es mínima o de aplicabilidad limitada. En el siguiente gráfico, se agrupan una serie de herramientas (asociadas a distintos recursos) (Gráfico $\mathrm{n}^{\mathrm{0}} 4$ ) que facilitan la labor educativa del docente de cara a "mejorar la lectoescritura, el desarrollo de habilidades básicas e inteligencia (la atención, la estimulación cognitiva, la estimulación del lenguaje o el razonamiento lógico-matemático)" (28:65, 23:25, D28). 
Gráfico 4 - Recursos y herramientas para trabajar la atención a la diversidad.

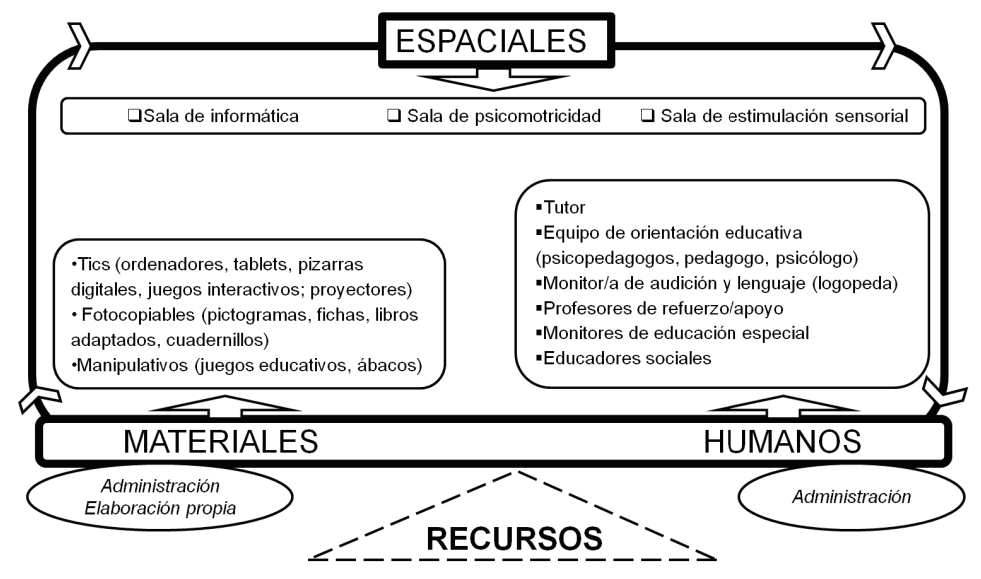

Por último, se analizan las respuestas sobre metodología y resultados de evaluación. En relación a cómo se evalúa, el profesorado coincide en la existencia de un protocolo de actuación con el estudiante que abarca diferentes fases: "se parte de una evaluación inicial presentando un informe al equipo docente; se desarrollan los distintos programas específicos de intervención; se analizan las tareas durante las distintas sesiones anotando las dificultades, avances...etc., que darán lugar a una reestructuración de los mismos; se termina con un nuevo informe individualizado, recogido dentro de la memoria final de curso, para comprobar la evolución progresiva del alumno" (42:59, 73:85, D42).

Este es un proceso constituido por un número indefinido de estrategias y diseños, tales como "la observación directa, pruebas escritas y orales" (20:58, 38:39, D20); "la valoración de su actitud dentro del aula" (41:67:68, D41); "diarios de clase, trabajos y puesta en práctica en clase, tareas para casa” (13:76, 49:51, D13), que dependerá de las peculiaridades de los escolares y de su intervención, y al que se le atribuye como características esencial "un desarrollo continuo" (24:68, 47:47, D24) durante el curso y en el que tutor y el equipo docente cobran especial relevancia; además, algunos destacan el logro de resultados más positivos cuando intervienen otros agentes que ayudan a dar un sentido más amplio a la evaluación: "en los casos en los que existe una buena coordinación de familias, tutores y especialistas los resultados son muy buenos" (39:76, 34:34, D39).

El aula

Una segunda dimensión engloba diferentes factores que intervienen en esta y, que por tanto, son de especial interés para analizar el impacto que pueden generar en la atención a la diversidad del alumnado.

La organización del aula se estudia con el objetivo de analizar los detalles más relevantes del trabajo del profesorado de PT y si los espacios se distribuyen en función de los aprendizajes. En primer lugar, destaca la unanimidad existente en estrategias ligadas a las necesidades del alumnado así como el apoyo en la figura docente: 
"es muy importante crear vinculos emocionales entre nosotros/as y los/as niños/as para que se sientan seguros/as, y comprometidos" (8:55, 76:79, D8). A partir de esta concepción, se describen varias estructuras de distribución de aula (priorizándose los grupos cooperativos) (Gráfico n ${ }^{05}$ ): la mesa del profesorado y los ordenadores pueden o no tener cabida; la división por rincones asociados a centros de interés y a las necesidades educativas son las más frecuentes; y los materiales se almacenan en armarios y/o estanterías.

Gráfico 5 - Modelos de distribuición del aula para atender a la diversidad.
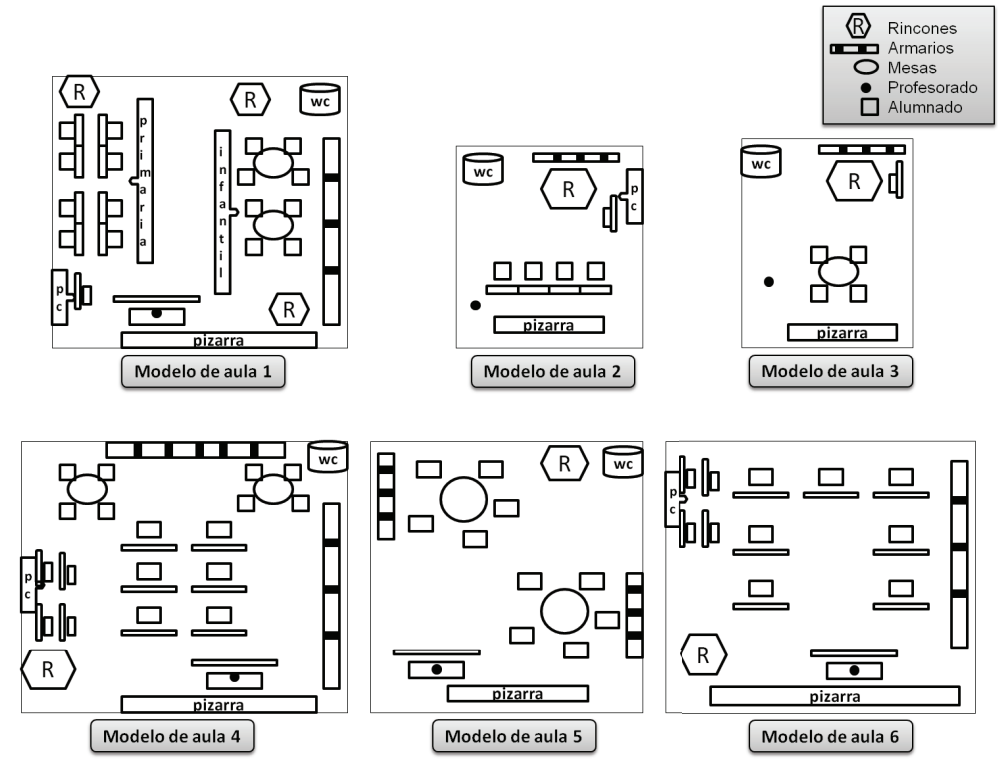

Otro factor relevante es el clima generado en el aula. El profesorado mantiene que han de crear unas condiciones favorables para una correcta intervención y un fructífero proceso de enseñanza y aprendizaje ("Un clima de confianza y respeto, no amenazante y facilitador de interacciones comunicativas será el objetivo de mi papel como maestro de apoyo. Valoraré las aportaciones y progresos del alumnado procurando potenciar un autoconcepto positivo -pedagogía del éxito-") (46:49, 77:79, D46). A partir de ahí, evidencian que los estudiantes se sienten motivados y están cómodos en el aula. También apuntan que el resto de profesores se posicionan positivamente en las relaciones (" $\mathrm{La}$ relación entre los diferentes profesionales se ve que es muy buena y el clima en el aula igual, ya sea entre los alumnos y entre la educadora y la profesora de educación especial') (3:45, 87:88, D3).

Para finalizar, se incide en la participación de la familia. En concreto, la opinión generalizada de todos los informantes gira en torno a la siguiente idea: "Hay familias muy implicadas que favorecen la mejora de los alumnos, familias que trabajan mano a mano con el profesorado, y otras que se desentienden por completo, es más, perjudican gravemente la situación, desatendiendo a sus propios hijos. Y resulta que el entorno familiar es un factor clave para la mejora de alumnado" (29:66, 85:87, D29). 
En las familias implicadas, su participación se asocia a una tutoría periódica cada 3 o 4 semanas junto a los profesionales que intervienen en la educación del estudiante y en la que se orienta a los padres en el trabajo en casa y se informa del proceso que sigue y a la asistencia al centro/aula para desempeñar funciones en diversas actividades propuestas ("programas especificos de conducta; talleres de cocina o de animación a la lectura; acompañamiento a salidas extraescolares; etc.") (40:65, 76:78, D40); a las menos implicadas se le atribuyen diversos factores como causantes de esta ausencia prolongada en la vida del aula y del centro ("no reconoce tanto la enfermedad de su niño"; "situaciones familiares complicadas"; "se sienten perdidas y a veces frustradas"; "Solo interesan los resultados y además, no realizan ninguna propuesta de mejora”) (33:58, 94:97, D33).

Avances y limitaciones

Se realiza una panorámica sobre las medidas diseñadas para conseguir una escuela para todos, sin exclusiones, señalando los logros alcanzados y los obstáculos vigentes.

En relación a las medidas adoptadas emergen argumentos a modo de principios:

- "Incluir al alumnado en todas las actividades, tanto a nivel de centro como de su grupo clase, poniendo a su disposición los recursos que necesite e intentando que sea un alumno más con sus particularidades" (1:74, 123:124, D12).

- "Adaptarse a las necesidades de cada alumno para que lleguen al nivel esperado por medio de adaptaciones curriculares significativas y no significativas" (7:63, 109:111, D7).

A partir de estos, se realiza una síntesis de las principales medidas que como profesionales promueven:

a) "Desarrollar en las aulas (en determinados momentos) un trabajo cooperativo basado en el programa Aprender para Cooperar y Cooperar para Aprender" (37:45, 98:99, D37).

b) "El apoyo dentro del aula por parte de un segundo profesor y la atención totalmente coordinada entre tutores y especialistas" (10:87, 147:147, D10).

c) "Una vez al año se realiza una semana para trabajar la diversidad e ir sensibilizando al alumnado" (5:60, 118:119, D5).

d) "Disponemos de un plan de convivencia para mejorar las relaciones dentro del centro" (2:45, 133:134, D2).

e) "Trabajar en grupos heterogéneos, se dedica una hora semanal para realizar dinámicas de grupos y fomentar habilidades sociales y cambios de roles" (9:31, 89:91, D9).

f) "Metodologías activas basadas en la neuroeducación; programación flexible, dinámica y menos directiva; implantación del trabajo por proyectos; aprendizaje basado en sus intereses; tertulias dialógicas, etc.” (41:75, 197:199, D41). 
Todas estas inciden tanto en las programaciones, donde se precisan las actuaciones a llevar a cabo, así como los horarios y tiempos destinados a atender a la diversidad, u otros aspectos: "mejora del clima del centro"; "comunicación con las familias, concienciación del claustro, normas de convivencia claras, la mediación entre alumnos, cambios metodológicos" (26:97, 233:239, D26).

También se pide una reflexión en torno a varios indicadores fundamentales para apreciar los resultados derivados del trabajo diario del docente en la atención a la diversidad. Destaca una alta satisfacción personal ("ves que los alumnos cada día dan un pasito más y superan algo más su dificultad" (23:48, 113:114 D23) o "siente ese cariño y esa protección de sus maestros y asi te lo hace ver, es una de las cosas más bonitas que puede ocurrir") (21:79, 153:155, D21); Las expectativas marcadas, son muy elevadas en la gran mayoría y se relacionan con "poder alcanzar la inclusión, al menos en el aspecto social y asertivo, con grupos de su misma edad, sin desarrollar ira, impotencia o agresividad" (14:97, 193:200, D14), o "ver que se sientan guiados y motivados mientras aprenden" (18:76, 103:105, D18); y finalmente, los logros alcanzados se asocian con "llevar a cabo una labor de la forma más normalizada posible, en la que el alumno aumente su confianza en si mismo, valore sus logros y acepte que todos tenemos una forma de aprender y sacarle el máximo partido a nuestras posibilidades" (32:75, 98:101, D32).

En la última categoría, se sintetizan (agrupados en tres bloques y por orden decreciente de prioridad) algunos obstáculos que el PT encuentra en su día a día (Gráfico no 6):

Gráfico 6 - Obstáculos que encuentra el profesorado al trabajar la diversidad.

\begin{tabular}{|c|}
\hline EL PROFESORADO Y LAS FAMILIAS: \\
\hline $\begin{array}{l}\text { 1o_ "El poco interés de algunos docentes, lo que implica poca coordinación entre PT y tutor o profesor del } \\
\text { área" (31:75, 88:88, D31). } \\
\text { 2o- "El anclaje metodológico de los docentes que no progresa hacia métodos más activos e inclusivos" } \\
\text { (19:59, 108:109, D19). } \\
\text { 3o_ "Familias sobreprotectoras/permisivas o que no asisten al centro" }(3: 91,178: 178, D 3) \text {. }\end{array}$ \\
\hline
\end{tabular}

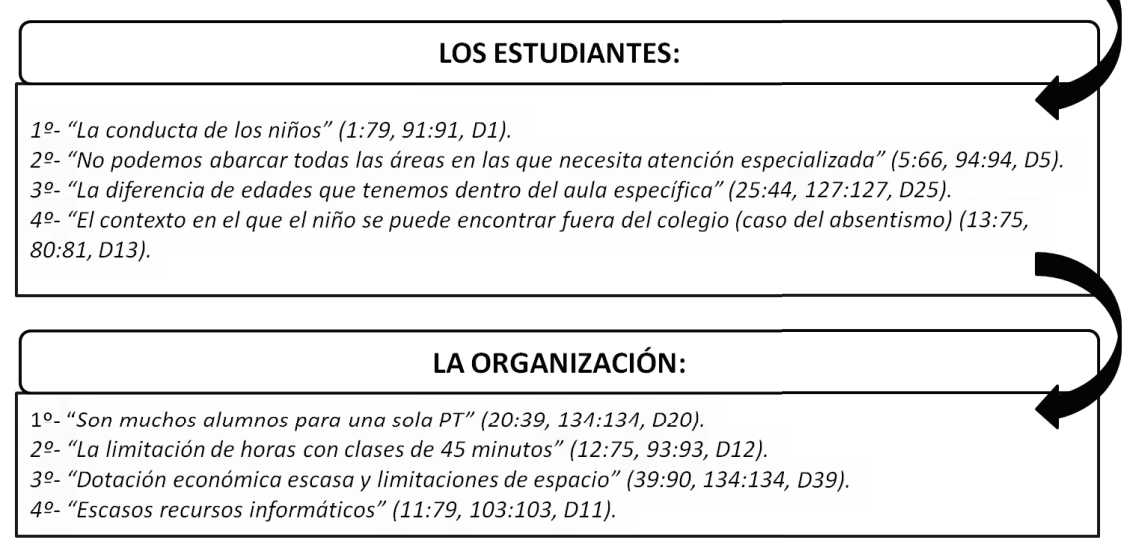

Aún son múltiples y sustantivos para alcanzar una práctica inclusiva que erradique las acciones segregadoras que aún perviven dentro de las aulas del S.XXI. 


\section{Discusión y conclusiones}

Con los objetivos planteados y los resultados obtenidos, se establece este proceso que muestra las concepciones de los profesionales de PT relativas a la diversidad, apoyadas en 3 contextos concretos:

\section{La intervención docente}

La atención a la diversidad como ámbito de trabajo del especialista en PT encuentra un obstáculo relevante al no ser reconocida por el resto de la comunidad docente, lo cual determina el inicio de procesos colaborativos necesarios (CRAWFORD y PORTER, 2004; SANDOVAL, SIMÓN y ECHEITA, 2012) y difumina su labor "dejando de ser el contrafuerte que se apoya en la pared maestra y se dedica a tapar agujeros" (JARQUE, 2016, p.44).

La concepción del PT sobre su intervención viene condicionada por las características del alumnado (CAMPO-MON et al., 2010) pero se visualiza la coexistencia de modelos apoyados en metodologías tradicionales (que frenan la búsqueda de una escuela más inclusiva) y modelos con metodologías más inclusivas (el trabajo cooperativo-interactivo) (BOOTH y AINSCOW, 2002). Además, en este proceso, reconoce la necesidad de una mayor implicación por parte de otros agentes (familia, tutores y equipos docentes) con el fin de construir un proceso compartido (HERNÁNDEZ et al., 2016; LLEDÓ y ARNAIZ, 2010).

Actualmente, la formación de este especialista es suficiente, aunque mejorable (CASANOVA, 2011), dado que las situaciones-problemas evolucionan a medida que lo hace la sociedad (problemas conductuales y asociados a la atención al déficit) (CEJUDO et al., 2016); de ahí que este proceso se complemente con las experiencias profesionales, factor que le permite gestionar recursos y crear otros que cubran las necesidades a las que la administración no responde con la concreción que se demanda.

\section{El funcionamiento del aula específica o de apoyo}

El aula específica se convierte en un recurso ampliamente utilizado (SANDOVAL, SIMÓN y ECHEITA, 2012; LLEDÓ y ARNAIZ, 2010) dada la amplia ratio de estudiantes con NEAE en el aula ordinaria y la falta de recursos personales para atender a estos alumnos junto a su grupo de referencia. Los especialistas en PT diversifican su estructura y organización (CAMPO-MON et al., 2010) para mantener una dinámica de aula que favorezca la interacción constante (en la medida de lo posible) con los aprendizajes abordados en el aula ordinaria, al tiempo que la implicación del resto del profesorado se vuelve imprescindible (PEARPOINT, 2015).

No obstante, quedan importantes labores que realizar respecto a la participación y el compromiso familiar con el centro para poder abordar diversas barreras ante una completa inclusión del los escolares (ESCARBAJAL et al, 2012). 


\section{Los avances y limitaciones}

El trabajo del PT en los centros educativos se sirve de una serie de medidas (metodologías activas basadas en la neuroeducación, grupos cooperativos, tertulias dialógicas, etc.) altamente positivas para el desarrollo de escuelas inclusivas y con las que se sienten satisfechos. Sin embargo, prosiguen demandas tradicionales, como la necesidad de lograr una escuela democrática (CALDERÓN y ECHEITA, 2016; TOBOSO et al., 2012) en la que se implique toda la comunidad educativa, el anclaje pedagógico de los docentes (ECHEITA, 2011; LLEDÓ y ARNAIZ, 2010) o la mejorable dotación de recursos en los centros educativos y, emergen otras más actuales, como la sobrecarga de funciones provocadas por la situación socioeconómica actual o el descenso en la consideración y reconocimiento social del docente (ESCARBAJAL et al., 2012), que dificultan la inclusión.

En definitiva, la idea que prevalece en la percepción del profesorado de PT es la de entender "la escuela como centro del cambio" (MURILLO et al., 2010) y para ello, la reflexión sobre la práctica y la exposición de evidencias contrastables (AINSCOW, 2011; LÓPEZ, ECHEITA y MARTIN, 2010) son estrategias que permiten al docente visualizar más allá de su acción pedagógica y creer en la posibilidad de construir una escuela inclusiva, donde la diversidad se atienda como un valor que enriquece el aprendizaje de todos y para todos, como indican los resultados del proyecto Teacher Education for Inclusion (EADNEE, 2012).

\section{Referências}

AINSCOW, M. Desarrollo de escuelas inclusivas. Ideas, propuestas y experiencias para mejorar las instituciones escolares. Madrid: Narcea, 2004. 308p.

Garantizar que cada alumno es importante: la mejora de la equidad dentro de los sistemas. En GAIRIN J.; ANTÜNEZ, S. (Eds.). Organizaciones educativas al servicio de la sociedad. Madrid: Volters Kluwer, 2010. V.1. p.261-282.

Respondiendo al desafío de la equidad en los sistemas educativos. Aula, v. 17, p. 73-87, 2011. Disponível em: <https://gredos.usal.es/jspui/handle/10366/120846>.

Haciendo que las escuelas sean más inclusivas: lecciones a partir del análisis de la investigación internacional. Revista de Educación Inclusiva, v.5, n.1, p. 39-49. 2012.

AINSCOW, M.; BOOT, T.; DYSON, A. Inclusion and the Standards Agenda: Negotiating Policy Pressure in England. International Journal of Inclusive Education, v. 10, n. 4, p. 295308. 2006. Disponível em: <http://dx.doi.org/10.1080/13603110500430633〉.

ALCALÁ DE GUADAIRA. Centro de profesores. Plan de formación. 2016. Disponível em: $<$ http://www.juntadeandalucia.es/educacion/portals/web/cep-alcala-guadaira/plan-de-centro . Acceso: 10 mar. 2016.

ALONSO, M.J.; ARAOZ, I. El impacto de la Convención Internacional sobre los Derechos de las Personas con Discapacidad en la legislación educativa española. Madrid: Ediciones Cinca, 2011.195p.

ALVAREZ, E. ET AL. Visión de los especialistas en pedagogía terapéutica de la integración del alumnado inmigrante. Aula abierta, v.37, n.1, p.57-66. 2009.

ANGULO, M.C. et al. Manual de servicios, prestaciones y recursos educativos para el alumnado con necesidades específicas de apoyo educativo. Sevilla: Junta de Andalucía, Consejería de Educación, 2008. 82p. 
ARNAIZ, P.; GUIRAO, J. M. La autoevaluación de centros en España para la atención a la diversidad desde una perspectiva inclusiva: ACADI. Revista Electrónica Interuniversitaria de Formación del Profesorado, v. 18, n. 1, p. 45-101. 2015. Disponível em: <DOI: http://dx.doi. org/10.6018/reifop.18.1.214341>.

BARTON, L. Estudios sobre discapacidad y la búsqueda de la inclusividad. Observaciones. Revista de Educación, n. 349, p. 137-152. 2008. Disponível em: <www.revistaeducacion.mec. es/re349/re349.pdf>.

BOOTH, T. El uso del Index for Inclusion en Inglaterra. En GINE, C.; DURAN, D.; FONT, J.; MIQUEL, E. (Coords.). La educación inclusiva. De la exclusión a la plena participación de todo el alumnado. Barcelona: Horsori, 2009. p.143-160.

CALDERÓN, I.; ECHEITA, G. Desafíos ineludibles para la construcción de escuelas inclusivas. Dossier GRAÓ, n.1, p.35-41.2016.

CAMPO-MON, M.A. et al. Funcionamiento de la integración en la enseñanza según la percepción de los maestros especialistas en Pedagogía Terapéutica. Psicothema, v. 22, n. 4, p. 797805. 2010.

CASANOVA, M. A. De la educación especial a la inclusión educativa. Estado de la cuestión y retos pendientes. CEE Participación Educativa, n.18, nov. 2011, p. 8-24. Disponível em: $<$ http://www.educacion.gob.es/revista-cee/pdf/n18-casanova-rodriguez.pdf>. Acceso: 19 oct. 2016

CASTILLEJA DE LA CUESTA. Centro de profesores. Plan de formación. 2016. Disponível em: <http://www.juntadeandalucia.es/educacion/portals/web/cep-castilleja-cuesta/plan-decentro>. Acceso: 10 mar. 2016.

CEJUDO, J. et al. Necesidades de formación de maestros de infantil y primaria en atención a la diversidad. Bordón, v. 68, n.3, p. 23-39. 2016.

CRAWFORD, C.; PORTER, G. Supporting Teachers: A Foundation for Advancing Inclusive Education. Toronto: L'Institut Roeher, 2004.23p.

EADNEE. Formación del profesorado para la inclusión en Europa. Desafíos y oportunidades. 2012. Disponível em: < http://www.european-agency.org/agency-projects/teacher-education-forinclusion>. Acceso: 21 jul. 2016.

ECHEITA et ál. Paradojas y dilemas en el proceso de inclusión educativa en España. Revista de Educación, n.349, p.153-178. 2009.

ECHEITA, G. El proceso de inclusión educativa en España. ¡Quien bien te quiere te hará llorar!, CEE Participación Educativa, n.18, nov. 2011, p.117-128. Disponível em: <http://www. educacion.gob.es/revistacee/pdf/n18-echeita-sarrionandia.pdf> . Acceso: 19 oct. 2016.

ECHEITA, G. et al. Educación inclusiva. Sistemas de referencia, coordenadas y vórtices de un proceso dilemático. En VERDUGO, M. Á.; SHALOCK, R. (Coords.). Discapacidad e Inclusión. Manual para la docencia. Salamanca: Amaru, 2013. cap. 14, p. 329-357.

ÉCIJA-OSUNA. Centro de profesores. Plan de formación. 2016. Disponível em: < http:// www.juntadeandalucia.es/educacion/portals/web/cep-osuna-ecija/plan-de-centro>. Acceso: 10 mar. 2016.

ESCARBAJAL, A. et al. La atención a la diversidad: la educación inclusiva. REIFOP, v.15, n.1, p. 135-144. 2012. Disponível em: <http://www.redalyc.org/pdf/2170/217024398011.pdf>. Acceso: 15 nov. 2016.

ESCUDERO, J.M.; MARTÍNEZ, B. Educación inclusiva y cambio escolar. Revista iberoamericana de educación, n. 55, p. 85-105. 2011.

FLORIAN, L.; BLACK-HAWKINS, K. Exploring inclusive pedagogy. British Educational Research Journal, n.37, p.813-828. 2011. Disponível em: <doi:10.1080/01411926.2010.5010 96>. 
GLAT, R.; PLETSCH, M.; FONTES, R. Educação inclusiva \& educação especial: propostas que se complementam no contexto da escola aberta à diversidade. Revista educação, Santa Maria, v. 32, n. 2, p. 343-356. 2007. Disponível em: <http://www.ufsm.br/ce/revista>. Acceso: 19 sept 2016.

HERNÁNDEZ, M. A. et al. Familia, inmigración y comunicación con el centro escolar: un estudio comparativo. Educación XX1, v. 19, n. 2, p. 127-151. 2016. Disponível em: <doi: 10.5944/ educXX1.14229>. Acceso: 09 ago. 2016.

JARQUE, J.M. ¿De qué hablamos, de inclusión o de escuela inclusiva? Dossier GRAÓ, n.1, p.42-46. 2016.

LEBRIJA. Centro de profesores. Plan de formación. 2016. Disponível em: < http://www.juntadeandalucia.es/educacion/portals/web/cep-lebrija/plan-de-centro>. Acceso: 10 mar. 2016.

LLEDÓ, A.; ARNAIZ P. Evaluación de las prácticas educativas del profesorado de los centros escolares: indicadores de mejora desde la educación inclusiva. REICE, v.8, n.5, p. 97-109. 2010. Disponível em: <http://www.redalyc.org/articulo.oa?id=55119084007>. Acceso: 13 mar 2016.

LÓPEZ-MELERO, M. La escuela inclusiva: una oportunidad para humanizarnos. Revista Interuniversitaria de Formación de Profesorado, v. 26. n. 2, p. 131-160. 2012. Disponível em: <http://www.redalyc.org/articulo.oa?id=27426890007>.

LOPES, J.A.; MONTEIRO, I.; SIL, V.; RUTHERFORRD, R.B.; QUINN, M. M. Teachers' perceptions about teaching problem students in regular classrooms. Education \& Treatment of Children, v. 27, n. 4, p.394-419, 2004. Disponível em: <10.1177/1098300708330879>.

LÓPEZ, M.; ECHEITA, G.; MARTÍN, E. Dilemas en los procesos de inclusión: explorando instrumentos para una comprensión de las concepciones educativas del profesorado. Revista Latinoamericana de Educación Inclusiva, v.4, n.2, p.155-176, 2010.

LORA DEL RÍO. Centro de profesores. Plan de formación. 2016. Disponível em: <http:// www.juntadeandalucia.es/educacion/portals/web/cep-lora-rio/plan-de-centro>. Acceso: 10 mar. 2016.

MALDONADO, A. et al. Judgments of Teacher Training students from three European countries on the efforts of social institutions for the integration of immigrants. In: CUNNINGHAM, P. ; FRETWELL, N. (Eds.). Lifelong Learning and Active Citizenship. Londres: CiCe, 2010, p. 181-188.

MUNTANER, J. J. Prácticas inclusivas en el aula ordinaria. Revista de Educación Inclusiva, v.7, n.1, p. 63-79. 2014.

PEARPOINT, J. Inclusive Education, Person---Centered Planning \& Systems Change Challenging Permutations and Opportunities. Revista Electrónica Interuniversitaria de Formación del Profesorado, número de revista, v.18, n.1, p. 35-44. 2015. Disponível em: <DOI: http:// dx.doi.org/10.6018/reifop.18.1.214331>. Acceso: 23 jul. 2016.

ROSE, R. Primary school teacher perceptions of the conditions required to include pupils with special educational needs. Educational Review, v. 53, n. 2, p. 147-56. 2001. Disponível em: $<$ http://dx.doi.org/10.1080/00131910120055570>.

SÁNCHEZ-TERUEL, D.; ROBLES-BELLO, M.A. Inclusión como clave de una educación para todos: revisión teórica. REOP, v.24, n.2, p. 24 - 36. 2013.

SANDOVAL, M.; SIMÓN, C.; ECHEITA, G. Análisis y valoración crítica de las funciones del profesorado de apoyo desde la educación inclusiva. Revista de Educación, n. extra., p.117137. 2012 .

SCHAPER, N. Prácticas y saberes de los maestros en la Educación Primaria. La diversidad como foco de atención. Revista educación inclusiva, v.3, n. 3, p.35-50. 2010. 
Manuel Delgado García - Francisco Javier García Prieto

SEVILLA. Centro de profesores. Plan de formación. 2016. Disponível em: < http://www.juntadeandalucia.es/educacion/portals/web/cep-sevilla/plan-de-centro>. Acceso: 10 mar. 2016.

TOBOSO, M. et al. Sobre la educación inclusiva en España: políticas y prácticas. Intersticios: Revista Sociológica de Pensamiento Crítico, v. 6, n. 1. p. 279-295. 2012.

* Professor doutor da Universidade de Sevilla, Sevilla, Andalucia, Espanha.

** Professor doutor da Uinversidade de Huelva, Huelva, Andalucia, Espanha.

\section{Correspondência}

Manuel Delgado-García - Avenida de Alemania, n. 98, 3. C. Código Postal: 21002 - Huelva, España.

E-mail: mdelgado25@us.es-fjavier.garcia@dedu.uhu.es

Recebido em 20 de dezembro de 2016

Aprovado em 03 de março de 2017 